\title{
DES FILAIRES DANS LE TUBE DIGESTIF
}

\section{Par C. DESPORTES}

On considère le plus souvent que les Filaridæ ne parasitent pas, à l'état adulte, le tube digestif de leur hôte. En effet, la vérification de spécimens ayant une semblable localisation, et primitivement désignés sous le nom de Filaria, a presque toujours montré qu'il s'agissait en réalité de nématodes appartenant à d'autres groupes. Il y avait cependant des exceptions et l'on pensait alors qu'il s'agissait d'observations erronées sur l'habitat du ver. C'est ainsi que Seurat écrivait en 1921, à propos de Litomosa (1) filaria (v. Bened.) (= Litosoma filaria, v. Bened.), donné par van Beneden comme parasite de la cavité gastrique de l'oreillard : "Cette filaire... vit dans la partie du mésentère attenante à l'intestin; on la trouve parfois accolée à l'estomac, ce qui explique l'erreur faite par van Beneden qui dit avoir rencontré l'unique individu mâle qu'il a vu dans l'estomac du Vespertilio auritus... ».

Depuis, les différentes récoltes de cette filaire ou des espèces voisines nouvellement décrites [ $L$. americana Mc Intosh 1932, L. chiropterorum Ortlepp 1932, L. wite Krepkogorskaja 1933 et $L$. burgosi de la Barrera 1926 (= Ackertia burgosi Vaz. 1934)] confirment le point de vue de Seurat : elles ont toutes été effectuées dans la cavité abdominale des hôtes.

Cependant, tout récemment, notre excellent ami, le Prof. J. Callot, nous faisait parvenir une dizaine de nématodes filariformes recueillis parfaitement vivants dans la lumière de l'intestin grêle de Myotis myotis (Bechstein), capturés à Volvic (Puy-de-Dôme).

La détermination de ces spécimens devait alors nous convaincre qu'il s'agissait de Litomosa filaria (v. Bened.).

Ainsi, il apparaît nettement que cette localisation, quoique assez rare, existe : Litomosa filaria est capable de vivre aussi bien dans le tube digestif que dans les séreuses, et l'observation de van Beneden est bien exacte. Ajoutons qu'il s'agit très vraisemblablement là de vers égarés qui ont traversé la paroi du tube digestif en passant

(1) Le nom de genre Litosoma van Beneden, préoccupé par un Hémiptère, a èté remplacé par Litomosa Yorke et Maplestone 1926.

AnN. de Parasirologie, T. XXI, Nos $3-4,1946$, p. 138-141. 
des séreuses vers la lumière; néanmoins, nous avons trouvé cet habitat de filaires suffisamment curieux pour le mentionner en quelques lignes.

Nous terminons cette note en donnant quelques précisions sur lả morphologie des exemplaires qui en ont été l'objet :

Mâle de 20 à $21 \mathrm{~mm} .4$; femelle de 25 à $27 \mathrm{~mm}$.

Corps cylindrique d'un diamètre de $90 \mu\left({ }^{7}\right)$ et $150 \mu$ (\$), effilé postérieurement et légèrement cleviforme en avant. Cuticule assez épaisse, de

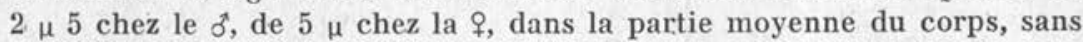
annulation visible. Champs latéraux peu larges $(q=75 \mu)$ avec une bande médiane hyaline de 8 à $10 \mu$ de largeur moyenne, qui s'étrécit $(5 \mu)$ à environ $550 \mu$ dc l'extrémité antérieure pour s'élargir de nouveau plus postérieurement. Pore excréteur, très petit (à $235 \mu$ de l'extrémité antérieure chez une femelle) ou non décelable; diérides non apparentes sur nos exemplaires. Ailes latérales absentes. Six papilles du cycle interne petites mais appréciables (fig. I-B) ; papilles submédianes du cycle externe et amphides non visibles (1). Lèvres nulles : ouverture buccale punctiforme ; cavitè buccale profonde cie 10 à $12 \mu$, à parois bien chitinisées et légèrement étranglée vers son milieu (fig. I-A). Esophage long de $415 \mu$ $\left(\delta^{2}\right)$ et $430 \mu($ (ᄋ), à portion glandalaire mal délimitée mais débutant en avant de l'anneau nerveux ; pas d'appareil valvulaire postérieurement. Anneau nerveux large, oblique (antéro-dorsal-postéro-ventral) à $175 \mu\left({ }^{2}\right)$ et $180 \mu$ (९) environ de l'extrémité antérieure. Il existe, un peu en arrière de l'anneau nerveux, deux bandes musculaires ventrales transversales qui se joignent sur la ligne médio-ventrale ( $\delta$ à $210 \mu$, $q$ à $230 \mu$ ) (fig. I-C).

Extrémité postérieure du mâle à 4 tours de spire. Queue fortement unciforme ; cloaque à $195 \mu$ de l'extrémité postérieure ; pas d'ailes caudales ; un petit mamelon (ou peut-être deux papilles contiguës) précloacal et médio-ventral ; deux paires post-cloacales sessiles ou faiblement pédiculées et une petite paire subterminale (fig., G). Phasmides non décelables. Spicule droit de 70 à $88 \mu$; gauche de 237 à $300 \mu$ (fig., H).

Testicule naissant à $700 \mu$ de l'extrémité antérieure et se prolongeant par le canal déférent au milicu du corps.

Extrémité postérieure de la femelle rectiligne ou presque, digitiforme, se terminant par un bourrelet asymétrique en arrière des champs latéraux, puis deux faibles saillies latéro-ventrales au voisinage desquelles on observe deux très petits orifices (= phasmides ?) (fig., D et E).

Anus situé à $140 \mu$ de la pointe caudale, large de 5 à $8 \mu$, mais estompé et vraisemblablement non fonctionnel chez ces femelles âgées.

Vulve petite, légèrement saillante, un peu en arrière de l'extrémité postérieure de l'œsophage (à $500-550 \mu$ de l'extrémité antérieure), sụivie du

(1) Chez L. americana, les deux cycles ont été observés par A. McIntosh et G. McIntosh (1935) ; cependant la figure de l'apex ne parait pas en rapport avec le texte : il semble en effet, d'après cette dernière, que l'espèce présente la disposition classique des 6 papilles au cycle interne et de 4 submédianes au cycle externe. 


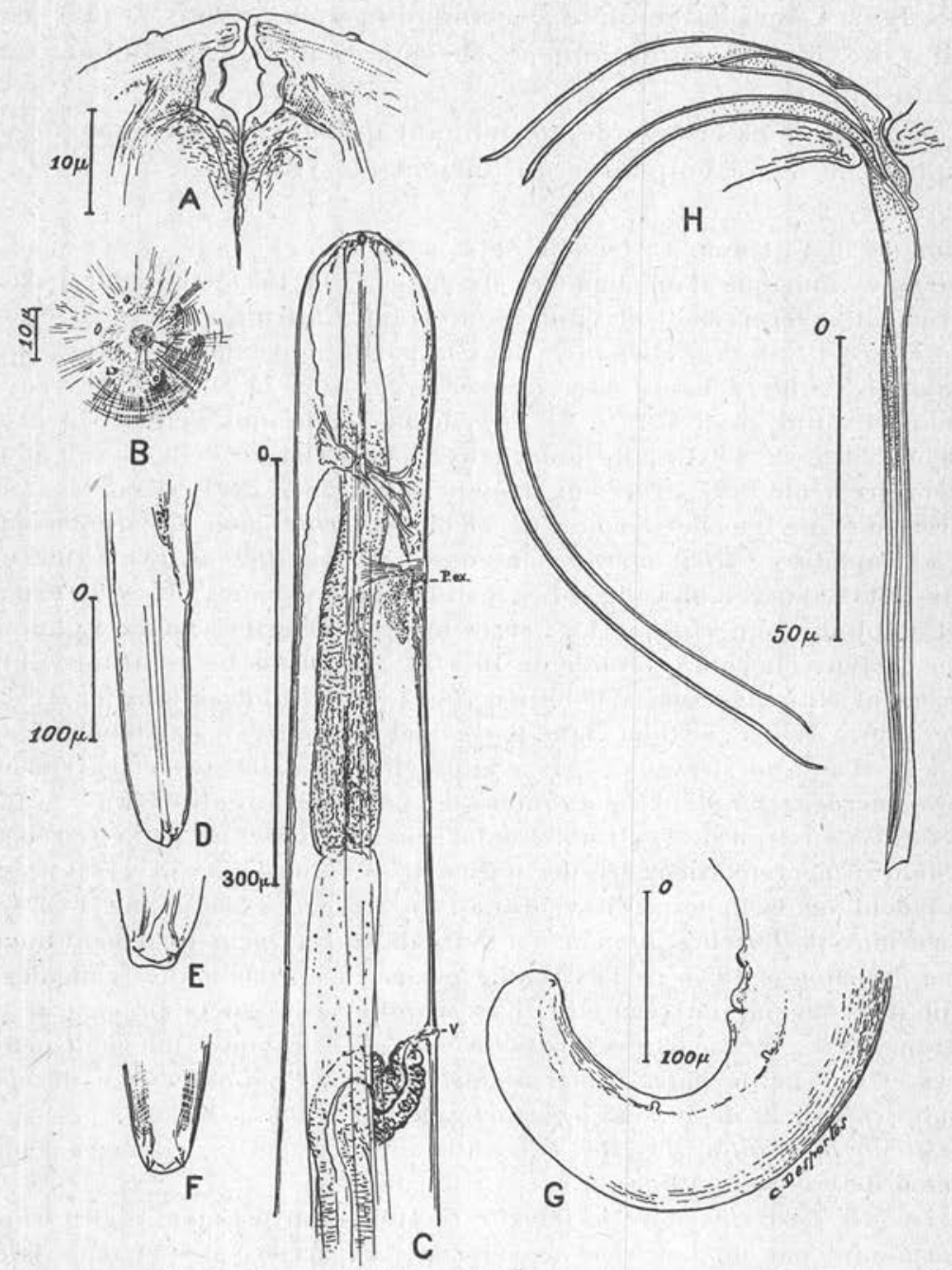

Fig. - Litomosa filaria (van Bened.) : A, capsule buccale ; B, vue apicale de l'extrémité antérieure ; C, partie antérieure du corps d'une femelle, vue de profil ; D, queue d'une femelle, vue latérale ; E, extrémité de la queue, vue ventrale; F, même extrémité, chez une femelle provenant de Plecotus auritus ; G, extrémité caudale du mâle ; H, spicules. (A, B, C, D, E, G et H, exemplaires provenant de Myotis myotis).

vestibule qui forme immédiatement un sphincter par un double coude en $S$, avant de se prolonger postérieurement, d'abord en une partie musculeuse et épaisse, de 600 à $700 \mu$ - à peu près rectiligne - puis en une seconde, à parois plus fines, de $2 \mathrm{~mm}$. environ de longueur; celle-ci 
s'unit alors à la trompe, bien plus large et qui court encore postérieurement sur une longueur de $800 \mu$ avant de se diviser en deux utérus. Opistodelphie. Oviductes de 700 et $1.100 \mu$ environ, en jonction avec les deux ovaires pelotonnés et refoulés à la partie postérieure, l'un étant situé en arrière de l'autre. QEufs utérins de $20 \times 15 \mu$. Embryons utérins de $140 \mu$.

Nous avons comparé ces exemplaires à ceux que nous avions recueillis à l'autopsie d'oreillards [Plecotus auritus (L.)] de Richelieu (Indre-et-Loire). Ces derniers se distinguent des premiers par leur taille inférieure, par la position de la vulve, toujours située à la limite postérieure de l'œsophage, par deux épines bien nettes à la queue des femelles (fig. I-F) et par la queue du mâle, seulement peu incurvée et dépourvue de papilles.

Ces caractères différentiels entre les Litomosa de Myotis myotis et de nos Plecotus auritus sont-ils suffisamment constants pour nous autoriser à voir là deux espèces distinctes? Nous ne pouvons actuellement répondre, car nous avons remarqué que les femelles des premiers contenaient toutes des embryons et celles de l'oreillard ne renfermaient que des œufs dans leurs utérus. Il se peut donc que ces différences constatées tiennent simplement à l'àge des vers.

Résumé. - Nous signalons la présence d'une dizaine de Litomosa filaria vivants dans l'intestin grêle de Myotis myotis. Il est donc vraisemblable que la localisation d'un de ces vers dans l'estomac d'un Plecotus auritus, signalée par van Beneden, était parfaitement exacte. Une description de ces spécimens, accompagnée de figures, est donnée.

\section{BIBLIOGRAPHIE}

Krepkogorskaja (T. A.). - Beitrag zur Fauna der Nematoden aus Rhombomys opimus Licht. aus Kasakstan. Zool. Anz., CII, 1933, p. 87-91.

McINTOSH (A.). - A new Filarid from the abdominal cavity of a Bat. Journ. of Parasit., XIX, 1932, p. 170.

McIntosh (A.) et McIntosh (G.). - Additional notes on two bat parasites, Dicrocclium lasiuri McIntosh, 1933 (Trematoda: Dicrocoliidæ) and Litomosa americana McIntosh, 1932 (Nematoda : Filariidæ). Proc. Helm. Soc. Washington, II, 1935 , p. 60-62.

Ortlepp (R. J.). - Some Helminths from South African Chiroptera. 8th. Rep. Veter. Serv. Pretoria, XVIII, 1932, p. 183-196.

Seurat (L.-G.). - Litosoma filaria Bened., type d'une nouvelle section de Filaires opisthodelphes. Bull. Mus. Hist. Nat., XXVH, 1921, pp. 103-106.

VAZ (Z.). - Ackertia gen. nov. for Litomosa burgosi de la Berrera 1926, with notes on the synonymy and morphological variations of Litomosoïdes carinii (Travassos, 1919). Ann. trop. Med. Parasit, XXVII, 1934.

Institut de parasitologie de la Faculté de médecine de Paris. Directeur : Professeur E. Brumpt. 